\title{
Research on Incentive Mechanism of Xinjiang Minority Staff
}

\author{
Yuan Ya, Zhang Chuanhui \\ College of economics and management, Tarim University, Alar, Xinjiang, 843300
}

Key words: Xinjiang minorities, Staff, Incentive measure.

\begin{abstract}
With the rapid development of social economy, the competition between enterprises becomes more and more intense. As a major resource of enterprise, the management of human resources in an enterprise determines its success or failure. Incentive mechanism is a major measure of human resources management, and how to use the incentive mechanism, give full play to the talent and retain talent, is related to the future development of an enterprise. Therefore, this paper studied the incentive mechanism of Xinjiang minority staff, analyzed the problems in this mechanism, and put forward the corresponding incentive measures, hoping to give full play to the talents in Xinjiang enterprises and promote the enterprises to develop well.
\end{abstract}

\section{Introduction}

It is pointed out in the 27 Opinions on Promoting the Development of Small and Medium Sized Enterprises in Xinjiang , formulated by Xinjiang Uygur Autonomous Government on September 9, 2010, that it is needed to strengthen the study of the modern management of the characteristic industries in Xinjiang, explore the way to improve the competitiveness of Xinjiang's characteristic industries, comprehensively improve the development of specialized personnel, the investment and management of human resources in the field of characteristic industries. On the one hand, as a newly starting group of enterprises, the minority enterprises in Xinjiang are weak in technological innovation ability and development capabilities, compared to those in other coastal developed areas of the country; on the other hand, the minority enterprises in Xinjiang lack capital, technology and high-level knowledge-oriented talents. Generally speaking, most of the value and profit of enterprises are created by staff. The staffs are the driving force for enterprises to grow, develop and gain sustainable competitive advantages. Therefore, how to develop and improve their potential and innovative enthusiasm is the most urgent task we are facing.

For most enterprises in Xinjiang, it is obvious that they still stay in the fields of national catering, traditional commerce and ethnic handicraft industry in the aspect of incentive measure. The enterprises are small in scale, single in production, low in science and technology and difficult in financing, which is not conducive to the development of minority enterprises; and one more important problem is that the comprehensive quality of the minority entrepreneurs is relatively weak, the operation ability of capital is poor, and the staff's cultural level is generally low.

\section{The importance of the application of incentive mechanism for minority staff in Xinjiang}

Employee incentive is to motivate and encourage staff through a certain means, and fully mobilize the enthusiasm and initiative of staff. As an important measure for the enterprises to retain talent and give full play to the talent, whether the incentive measures are effective related to management of enterprise human resources and the future development of enterprises. Especially for the enterprises, which can't retain staff in Xinjiang, the effective incentive mechanism is particularly important.

We see that in many large enterprises in Xinjiang, the enterprises' effective application of incentives in the staff management plays a very important role in stimulating the potential of staff, because the incentives make these minority staff devoted into the work actively, bring more performance for the enterprise, better promote the development of the staff and bring greater 
economic benefits for the development of enterprises.

The initiative and motivation in work are often directly related to employee productivity. Generally, effective incentives provide a better working environment for staff, and improve the enthusiasm and initiative of the staff, and then the efficiency of work will naturally increase. Therefore, it is very important to provide a good working environment for staff, use effective incentive measures to meet the needs of staff in improving efficiency of the staff, which reflects the business level of an enterprise, and relates to the development speed of an enterprise.

If work efficiency is a measure of quantity, then working ability is a measure of quality. Effective incentive measures not only play an important role in the improvement of work efficiency, but also play a very important role in improving their work ability. Because effective incentive measures can improve the enthusiasm of staff, and has an active effect on giving full play to the staff's talent, which largely reflects the work ability of staff.

The research shows that effective incentive measures can effectively reduce the loss of talent, improve the satisfaction of staff with enterprise, increase the sense of belonging of the staff, and create a good working environment. The harmony between staff and staff, staff and managers is very conducive to the harmonious development of enterprises.

\section{Analysis on incentive measures of Xinjiang minority staff}

At present, in Xinjiang minority enterprises, knowledge-oriented talent with bachelor degree or above accounted for about fifteen percent of the total number of staff. The total number of knowledge talents is small, and the rate of losing talent is relatively high. According to statistics, the flow rate of knowledge-oriented talent is as high as sixty percent, far higher than the rational flow rate of fifteen percent. The excessive flow of knowledge-oriented talent will not only restrict the development of themselves, but also become a acknowledged profit trap for enterprises, which is very unfavorable to the development of enterprises.

According to the analysis on the needs of staff of different age groups, we find that young people under 27 years old are at the stage of introduction of their careers, and they pay more attention on the company's complete training system, learning opportunities, financial security, growth opportunities and the work itself; middle aged staff are in the growth stage, they are more emphasis on development opportunities, interesting work, and participating in the management of the company; staff between 40-50 years of age are at the maintenance stage of their careers. They pay more attention to opportunities for advancement, empowerment and good working conditions, staffs over the age of 50 are in the decline stage of their career, they will pay more attention to honor incentives, knowledge sharing and harmonious interpersonal relations and other aspects of improvement.

\section{Matters need attention to the incentives of minority staff}

In the process of incentive, the enterprise should take the actual needs and expectations of minority staff into account, and take corresponding incentives according to their expectations. Only in this way can the expectations of the minority staff be satisfied, and retain the minority workers, and stimulate their enthusiasm and initiative in work.

Incentive is not only to meet the needs of the minority staff, the ultimate goal is to stimulate the enthusiasm of staff and improve work efficiency and work ability by means of incentive. Only in this way can the staff and the enterprises be able to develop while satisfying the needs of the minority staff. Any measure that can't motivate staff is simply to meet the needs of staff in temporary, and cannot really achieve the joint development of staff and enterprises.

Any incentives that do not meet the needs and expectations of staff, or even against the actual needs of staff, are ineffective incentives. Because enterprises must have a deeper understanding of the Xinjiang minority staff, grab their actual needs and expectations and adopt effective incentives to stimulate their activities and initiative.

During the implementation of the incentive, attention should be paid to the fairness, impartiality 
and openness of the incentives. The enterprise should enhance transparency in incentives, ensure that those who are motivated can enjoy the incentive treatment fair, impartially and openly, and build a transparent incentive mechanism for minority staff, so that they can display their talents and abilities under the encouragement of incentives.

\section{Suggestions on motivating Xinjiang minority staff}

Minority staffs want better jobs and higher incomes. But if there is not a strong enterprise to support, there is no development space for individuals. To retain staff, enterprises must draw up the development plan, solve the difficulties and obstacles, and achieve goals one by one. Culture is the soul of an enterprise, the excellent culture is endless. The enterprise should influence the staff with the corporate culture, and enhance their sense of mission. Only when we see the hope of enterprise development, staff can be more motivated, and the dreams of individuals will be realized eventually.

Minority staffs have higher material requirements for the working environment, and the office environment will affect people's mood, and then affect work efficiency. Therefore, it is very important to provide comfortable hardware environment for staff and provide the basic facilities to meet the needs of work. Computer, printing and copying equipment should run fast, safe and smooth, and the design of office should be reasonable. Facing the same work, everyone has their own advantages and disadvantages, the enterprise should advocate working in unity and helping each other in work and study to create a good working atmosphere. The environment has influence on the talents, and the internal soft environment of enterprise influences staff greatly, efficiency of work is closely related to the surrounding environment, a good working atmosphere will make people feel pleasure, and thus they will work actively. By creating a good internal work environment, the enterprise can improve their work efficiency and form a harmonious working atmosphere.

People have material needs, as well as spiritual needs. Enterprises should understand the situation of minority staff from the two aspects to meet their material needs and the spiritual needs. The enterprise should design scientific and reasonable salary system, assess the quantity of salary, find and improve problems. It can also increase the account of performance pay of the staff in the total wage, link the income of staff with enterprise benefits, and promote working together. If the enterprise operating efficiency is good, personal wages and bonuses will be high, and vice versa. Staff and enterprises form a community of interests, the development of enterprises related to their immediate interests, so that the enthusiasm of staff can be improved.

The enterprise should provide training opportunities for excellent minority staff, and enable staff to broaden their horizons, learn new knowledge and skills, and increase their abilities. For staff, they are still lacking knowledge and skills through practice, the young people are not rich in experience, the new staff is lack of experience, which cannot meet the requirements of normal work, so they will make all sorts of errors in their work. The enterprise should provide more opportunities to practice through the related training, and continue to assign tasks to young staff. Learning in practicing helps the staff to grasp the relevant knowledge and skills, complete the work, and promote the development of their own business. The enterprise can increase benefit and retain talent to win the future.

In the fierce market competition, enterprises should give priority to efficiency and give consideration to fairness. The staff should pay attention to the development space of the workplace, realize their own value, keep practical and rational. To elevate minority staff with capability is the encouragements to minority staff, which can make them work harder, provide the space for the minorities to display their ability, so as to achieve the purpose of excitation.

\section{Conclusion}

As an important resource for the development of enterprises, it is very important to give full play to talents of the staff effectively. And the incentive mechanism play an important role in 
arousing the talents, especially in the Xinjiang area where the loss of talents is serious, it is not only related to the development of minority staff, but also the actual economic interests of enterprises and even the overall development of Xinjiang to make full use of incentive mechanism and effective incentive measures,

\section{References}

[1] Zhang Li. Study on the Risk and Control Mechanism of Loss of Senior Management in Enterprises[J]. Business Management, 2009 (05)

[2] Li Lirong. Research on Employee Motivation Mechanism [J]. China Management Information, 20015 (12.)

[3] Nuerguli-Cartier. Analysis on Incentive Mechanism for Senior Managers of Minority Enterprises in Xinjiang [J]. Discovering Value, 2014 (09)

[4] Nuerguli·Cartier. Analysis of the Incentive Mechanism of Non-economic Reward for Minority Enterprises in Xinjiang: taking Urumqi as an example[J]. China Journal of Commerce, 2012 (11)

[5] Qian Hongming, Xia Hui. Study on the Incentive Problems of Small and Medium-sized Enterprises in Xinjiang. [J]. CO-Operative Economy \& Science, 2015 (09) 